\title{
Appositionsthrombus als Komplikation endovenöser Katheterverfahren (Post ablation thrombus extension [PATE])
}

\author{
N. Schäffer; I. Weingard; M. Kiderlen; A. Theodoridis; L. Schuler; N. Kriechenbauer; \\ K. Hartmann \\ Venenzentrum Freiburg, Freiburg
}

\begin{abstract}
Schlüsselwörter
Appositionsthrombus, EHIT, PASTE, PATE, endovenöse Therapie, Varikosis, Thrombose, Laser, RFA, Radiofrequenz
\end{abstract}

\section{Zusammenfassung}

Appositionsthromben sind mit einer Prävalenz von $0,2-6 \%$ eine nicht seltene Komplikation nach endovenösen Katheterverfahren. Mögliche Risikofaktoren wurden bislang noch nicht ausreichend untersucht, außerdem fehlen Standards für eine angemessene Prophylaxe und ein einheitliches Therapieschema. Wir haben eine retrospektive Analyse von 2015-2017 bezüglich der Prävalenz sowie möglicher Risikofaktoren für das Auftreten eines Appositionsthrombus nach endovenösen Verfahren im Venenzentrum Freiburg durchgeführt. Die in der Literatur beschriebenen Risikofaktoren wie, Gefäßdurchmesser> 7,5mm, simultane Miniphlebektomien sowie ein erhöhter BMI konnten nicht verifiziert werden. Die Stadieneinteilung und die Bezeichnungen des Appositionsthrombus

Korrespondenzadresse

Nina Schäffer

Venenzentrum Freiburg

Zähringerstr. 14

79108 Freiburg

E-Mail: schaeffer.nina@gmx.at im internationalen Gebrauch wie EHIT (endovenöse hitzeinduzierte Thrombose) und PASTE (post ablation superficial thrombus extension) sind irreführend, da diese Komplikation auch bei nicht-thermischen Verfahren auftreten kann und es sich um eine Thrombusextension ins tiefe Venensystem handelt. Daher empfehlen wir die modifizierte Bezeichnung PATE (post ablation thrombus extension). Ein PATE 0 entspricht einem planen Verschluss und ist somit ein Therapieerfolg. PATE I beschreibt eine Thrombusextension mit Einengung des Lumens der tiefen Vene bis zu 25\%, PATE II bis $50 \%$ und PATE III $>50 \%$. Eine therapeutische Antikoagulation empfehlen die Autoren ab PATE II mit einer Dauer bis zum Verschwinden des Appositionsthrombus.

\section{Keywords}

Post ablation thrombus, EHIT, PASTE, PATE, endovenous laser treatment, Laser, RFA, thrombosis, varicosis

Post ablation thrombi extensions as complication after endovenous catheter procedures (Post ablation thrombus extension [PATE]) Phlebologie 2018; 47: 93-101 https://doi.org/10.12687/phleb2417-2-2018 Eingereicht: 30. Januar 2018 Angenommen: 02. Februar 2018

English version available at: www.phlebologieonline.de

\section{Summary}

Post ablation thrombi extensions are with a prevalence of $0.2-6 \%$ a not uncommon complication after endovenous catheter procedures. Potential risk factors have not been adequately studied, and there is a lack of standards for appropriate prophylaxis and a consistent treatment regimen. We carried out a retrospective analysis of the prevalence and possible risk factors for the occurrence of an apposition thrombus after endovenous procedures in the Vein Center Freiburg, Germany in 2015-2017. The risk factors described in the literature, such as vessel diameter> $7.5 \mathrm{~mm}$, simultaneous mini-phlebectomies and an increased BMI could not be verified. The staging and designations of the apposition thrombus in international use such as EHIT (endovenous heat-induced thrombosis) and PASTE (post ablation superficial thrombus extension) are misleading as this complication may also occur in non-thermal procedures and the thrombus extension is into the deep venous system. Therefore, we recommend the modified name PATE (post ablation thrombus extension). A PATE 0 corresponds to a plan closure and is therefore a therapeutic success. PATE I describes thrombus extension with lumen narrowing up to $25 \%$, PATE II to $50 \%$, and PATE III> $50 \%$. The authors recommend a therapeutic anticoagulation from PATE II on with a duration until the disappearance of the apposition thrombus. 

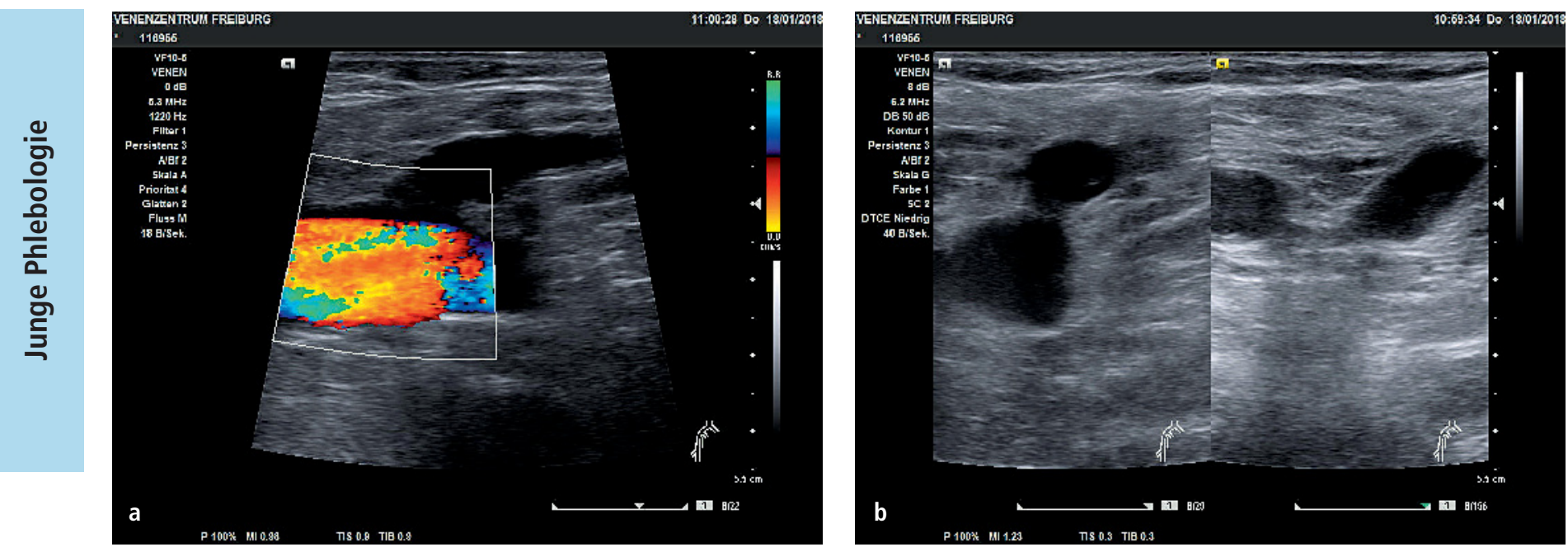

Abb. 1 a und b: Patient 0, eine PATE 0 zehn Tage nach Laser der Vena saphena magna (VSM) ist ein gewünschter Therapieerfolg erfolgt. Es zeigt sich eine Thrombusextension bis zur tiefen Vene und somit ein planer Verschluss der VSM.
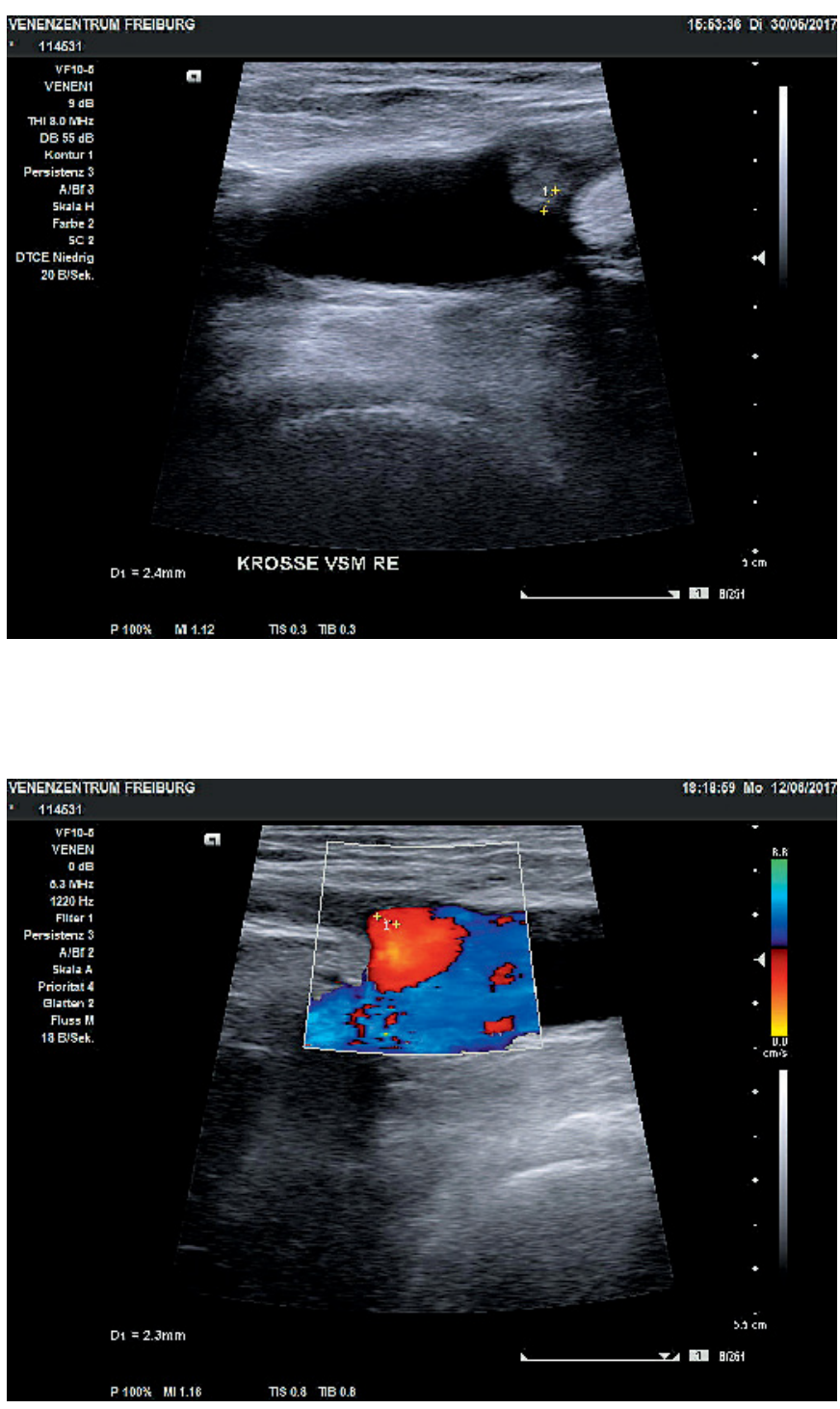

Abb. 2

Patient 1, PATE I zehn Tage nach Venefit ${ }^{\circ}$ der Vena saphena magna und Vena saphena accessoria anterior (VSAA) kombiniert mit einer Schaumsklerosierung rechts. Eine Miniphlebektomie wurde nicht durchgeführt.
Abb. 3

Patient 1, Nachkontrolle nach 12 Tagen. Der Thrombus war nicht mehr darstellbar, eine medikamentöse Therapie wurde nicht durchgeführt.
Endovenöse hitzeinduzierte Thrombosen (EHIT), oder besser auch postablative oberflächliche Thrombusextension (post ablation superficial thrombus extension (PASTE)) genannt, als Komplikation endovenöser Katheterverfahren werden laut aktueller Literatur bei $0,9 \%-6,0 \%$ der mit Laser,- und bei ca. 0,2\% der mit Radiowellenkatheter behandelten Patienten beobachtet und zeichnen sich durch ein appositionelles Thrombuswachstum in die tiefe Vene aus $(2,3,5,6)$. Die Bezeichnungen im internationalen Gebrauch sind leider irreführend, da es sich um eine Thrombusextension ins tiefe Venensystem handelt und nicht um eine superfizielle Extension. Daher empfehlen wir die modifizierte Bezeichnung PATE: post ablation thrombus extension (in Anlehnung an PASTE).

Als Risikofaktoren für das Auftreten einer PATE spielen gemäß aktueller Literatur vor allem große Gefäßdurchmesser $>7,5 \mathrm{~mm}$, simultane Phlebektomien sowie höhergradige CEAP Klassen eine große Rolle. Bezüglich des männlichen Geschlechts als Risikofaktor gibt es widersprüchliche Angaben in der Literatur $(5,6)$. In unserem Zentrum konnten diese Parameter nicht als Risikofaktoren verifiziert werden, auch ein erhöhter BMI, das gleichzeitige Behandeln der VSAA und die Dauer der postinterventionellen Antikoagulation scheinen keinen wesentlichen Einfluss auf das Entstehen eines PATE zu haben. $(\triangleright$ Tab. 4) Eine Risikoreduktion kann laut 

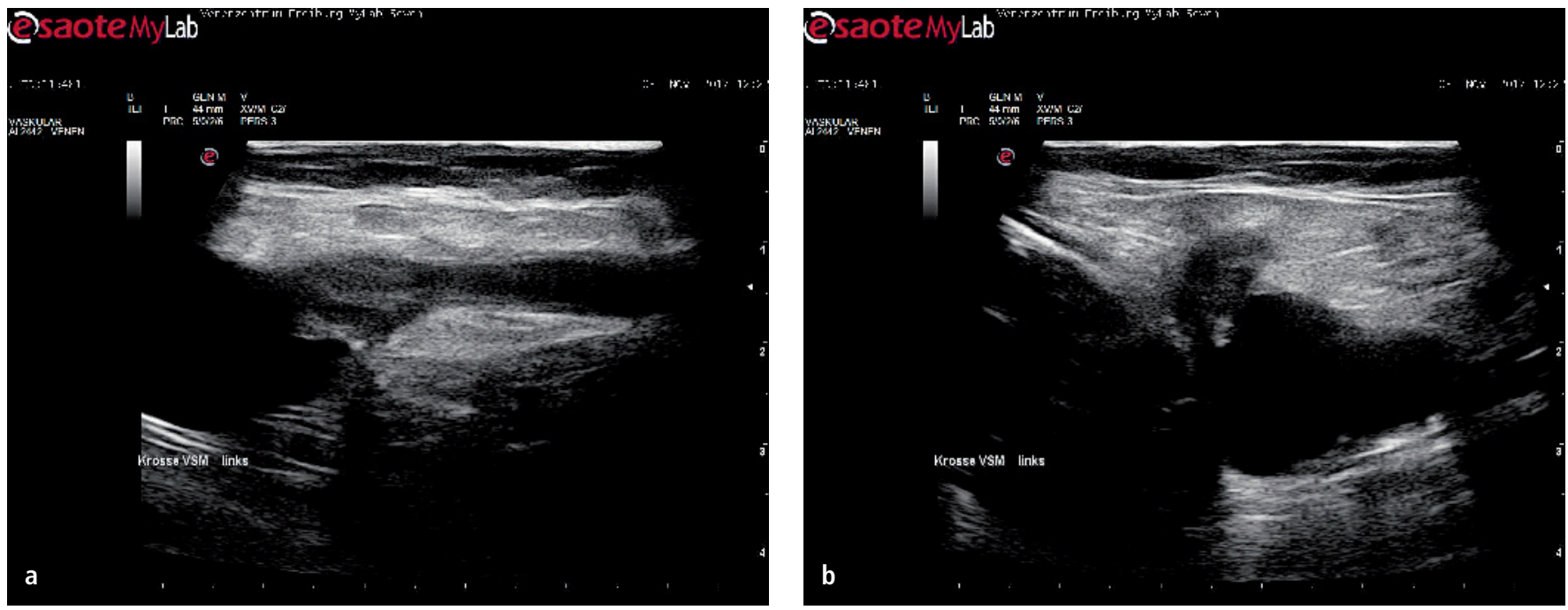

Abb. 4 a und b: Patient 2, PATE I links zehn Tage nach Laser der VSM beidseits (bds.) und Miniphlebektomie kombiniert mit einer Schaumsklerosierung. Eine Antikoagulation ist nicht erforderlich.
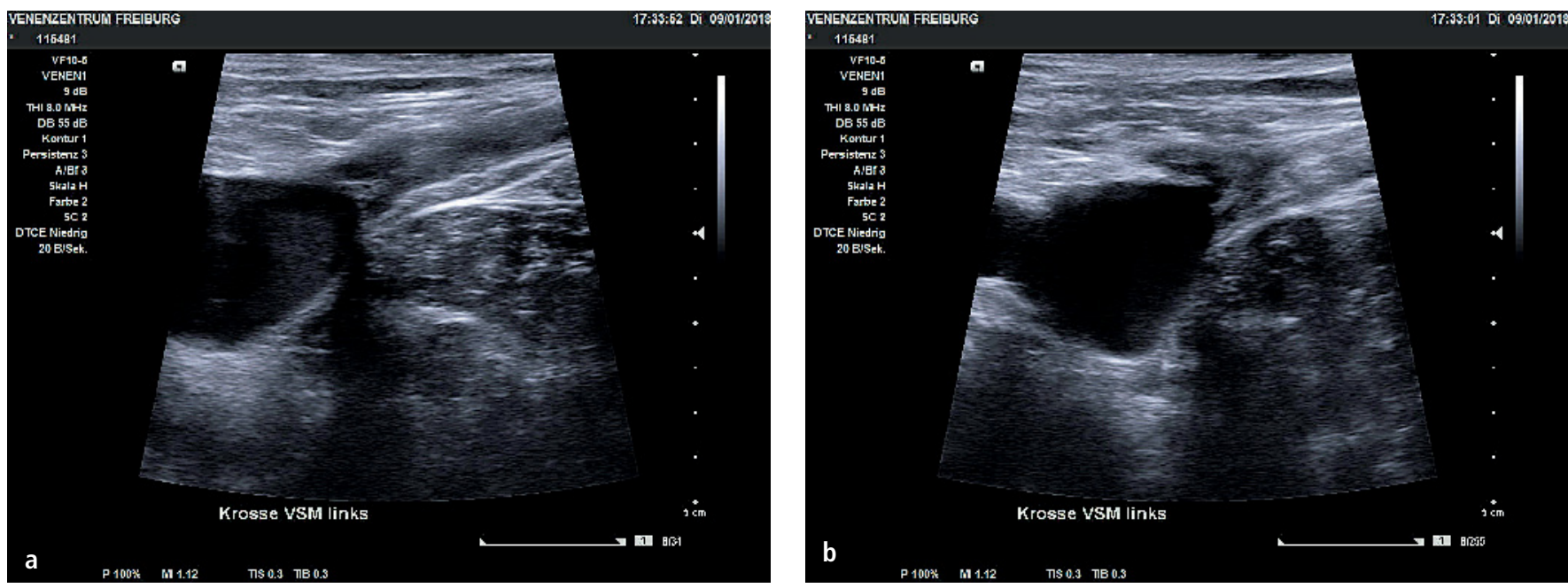

Abb. 5 a und b: Patient 2, Nachkontrolle nach zehntägiger Therapie mit Tinzaparin gewichtsadaptiert, der Thrombus war nicht mehr darstellbar und die Therapie mit dem NMH wurde nach 10 Tagen beendet.

Literatur durch den Einsatz von Lasersystemen im Wellenlängenbereich von 1470 $\mathrm{nm}$, welche ein hohes Absorptionsspektrum im Bereich des Wassers haben, erreicht werden $(2,5)$.

Insgesamt werden vier Schweregrade des PATE unterschieden $(1,4)$.

Modifiziert empfehlen wir folgende Einteilung:

- PATE 0: Ausdehnung des Thrombus bis zur tiefen Vene (=planer Verschluss = gewünschter Therapieerfolg)

- PATE I: Ausdehnung des Thrombus wenige Millimeter in die tiefe Vene hinein mit Einengung des Lumens bis zu 25\%.
Abb. 6

Patient 3, PATE III zehn Tage nach Laser VSM und VSAA. Auf eine Schaumverödung und Miniphlebektomie wurde verzichtet. Eine Therapie mit Tinzaparin gewichtsadaptiert wurde eingeleitet.

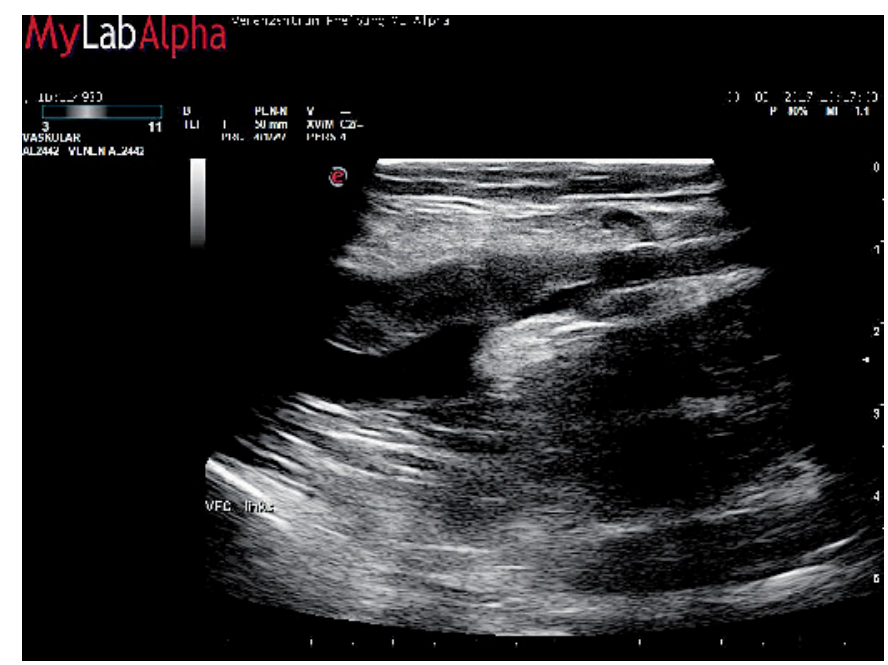



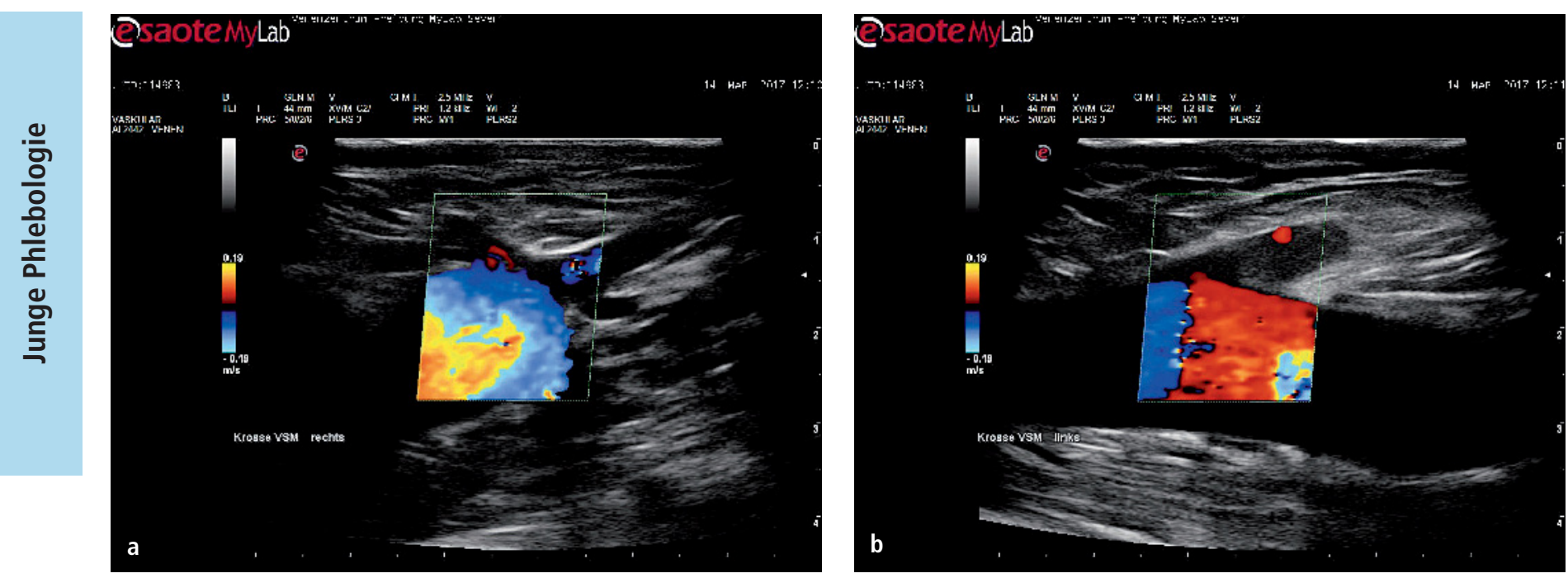

Abb. 7 a und b: Patient 3, Da sich der Appositionsthrombus nach 14 tägiger Therapie mit NMH noch nicht aufgelöst hatte wurde die Therapie mit Tinzaparin gewichtsadaptiert auf insgesamt 30 Tage verlängert. Danach war der Thrombus nicht mehr darstellbar und Tinzaparin konnte abgesetzt werden.
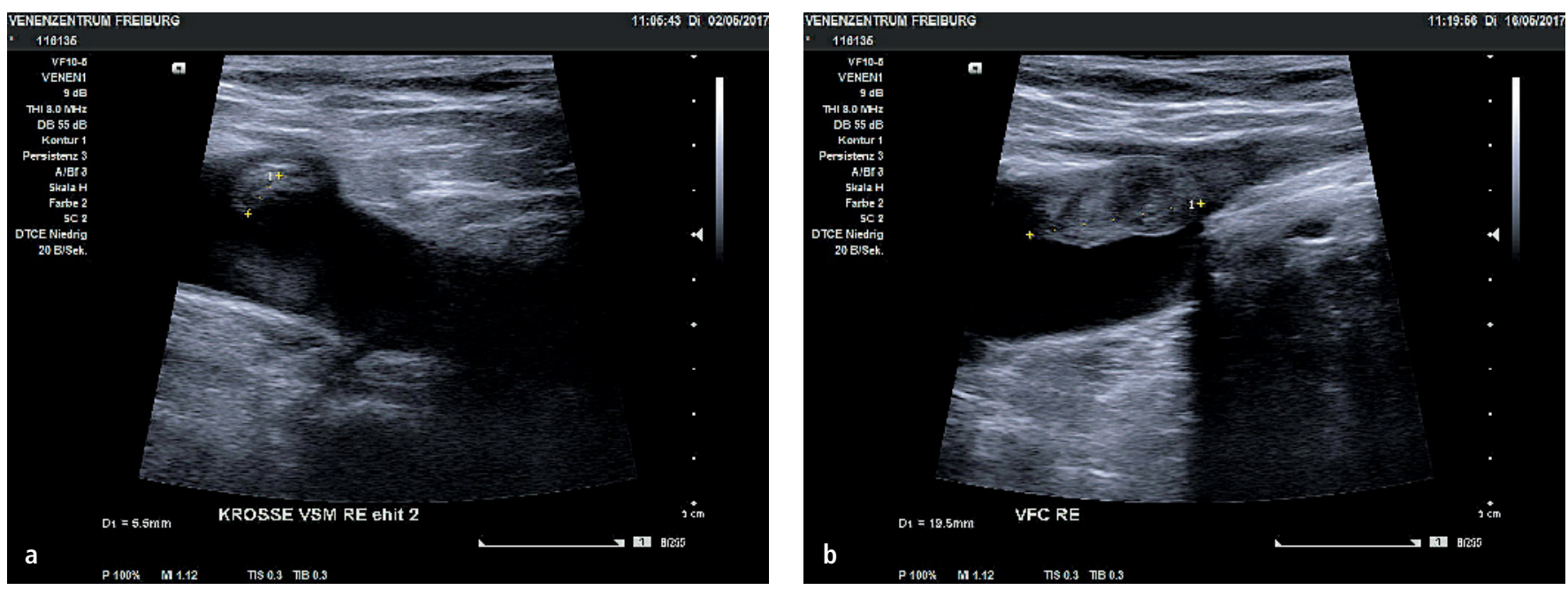

Abb. 8 a und b: Patient 4, PATE II rechts zehn Tage nach Laser der VSM bds. Kombiniert mit einer Schaumsklerosierung. Eine Therapie mit Tinzaparin gewichtsadaptiert wurde eingeleitet.

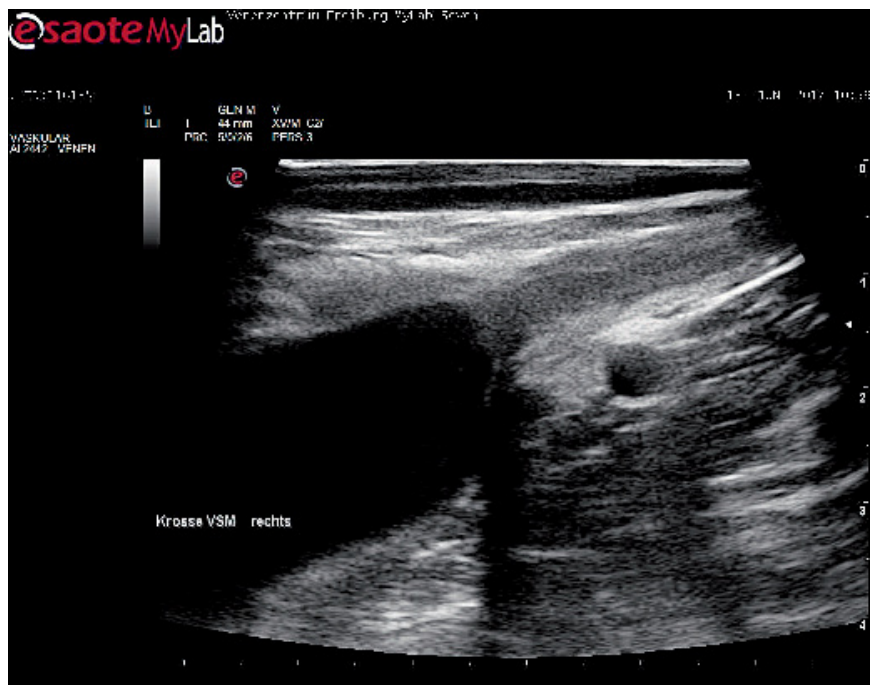

Abb. 9

Patient 4, Auch in diesem Fall hatte sich der Appositionsthrombus nach 14 tägiger Therapie mit NMH noch nicht aufgelöst, weshalb die Therapie auf insgesamt 30 Tage verlängert wurde. Anschließend war der Thrombus nicht mehr darstellbar und Tinzaparin konnte abgesetzt werden.
- PATE II: Ausdehnung in die tiefe Vene hinein mit Einengung des Lumens bis maximal $50 \%$

- PATE III: Einengung der tiefen Vene $>50 \%$

- PATE IV: vollständiger kurzstreckiger Verschluss der tiefen Vene

Ein PATE 0 ist ein gewünschter Therapieeffekt.

Ein PATE I wird innerhalb von ein- bis zwei Wochen nachkontrolliert bis sich die Vorwölbung in die tiefe Vene zurückgebildet hat, eine medikamentöse Therapie ist 

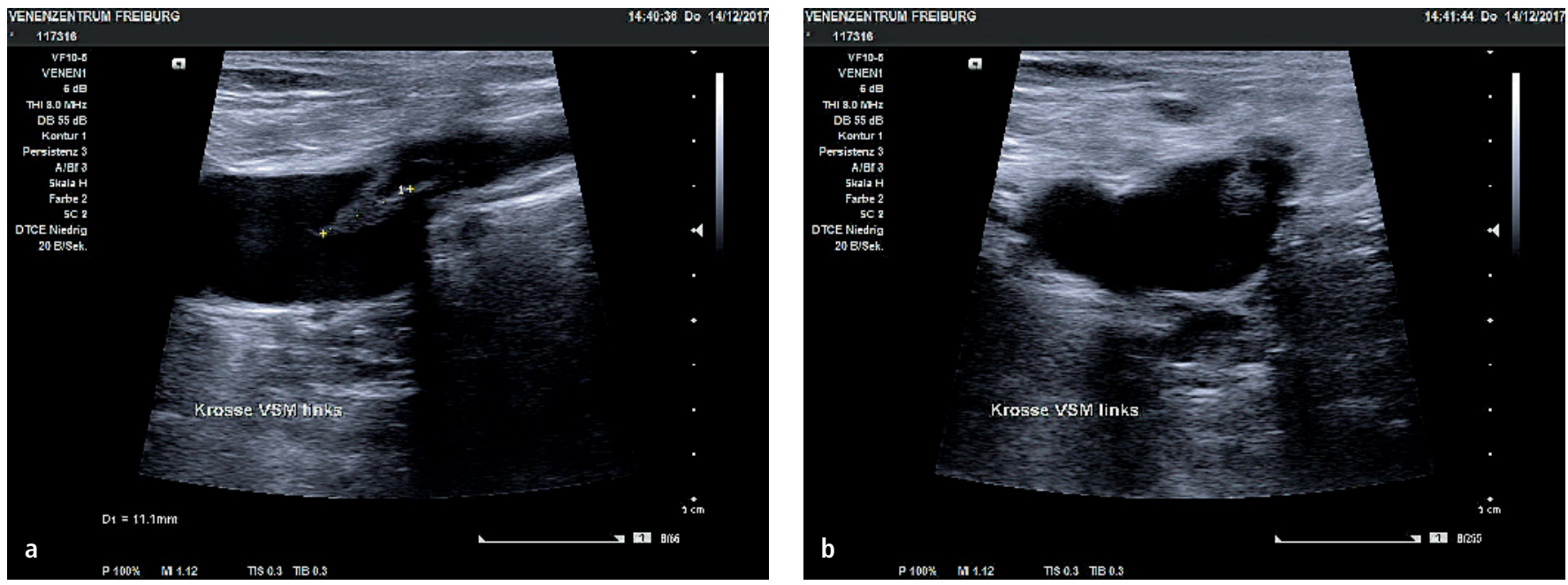

Abb. 10 a und b: Patient 5, fluktuierender (zapfenartiger) PATE I links nach Laser VSM und VSAA kombiniert mit einer Miniphlebektomie und Schaumsklerosierung. Eine Therapie mit Tinzaparin gewichtsadaptiert wurde eingeleitet.

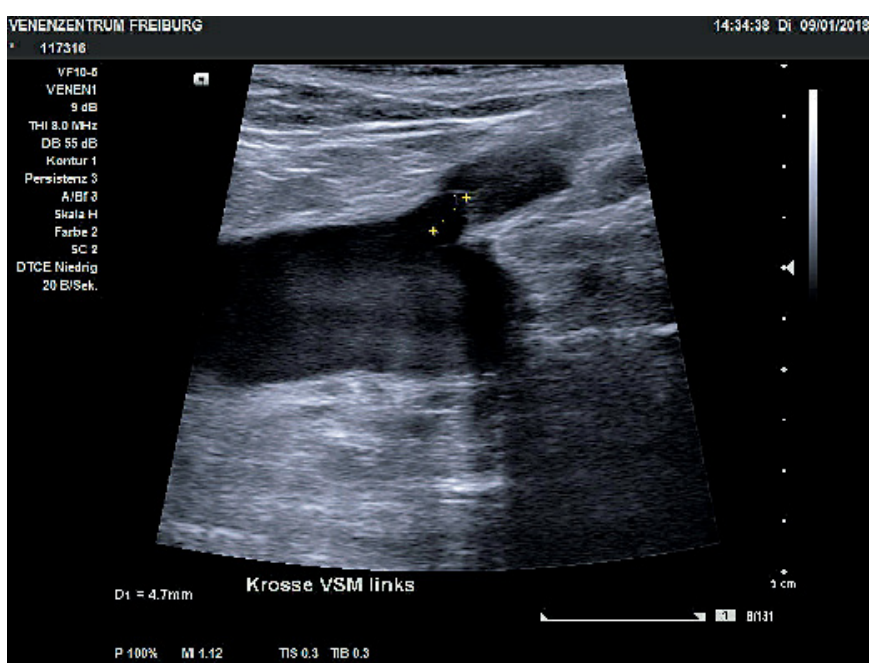

Abb. 11 Patient 5, Nachkontrolle nach 14 tägiger Therapie mit Tinzaparin gewichtsadaptiert. Der Thrombus hatte sich aufgelöst, das NMH wurde abgesetzt.

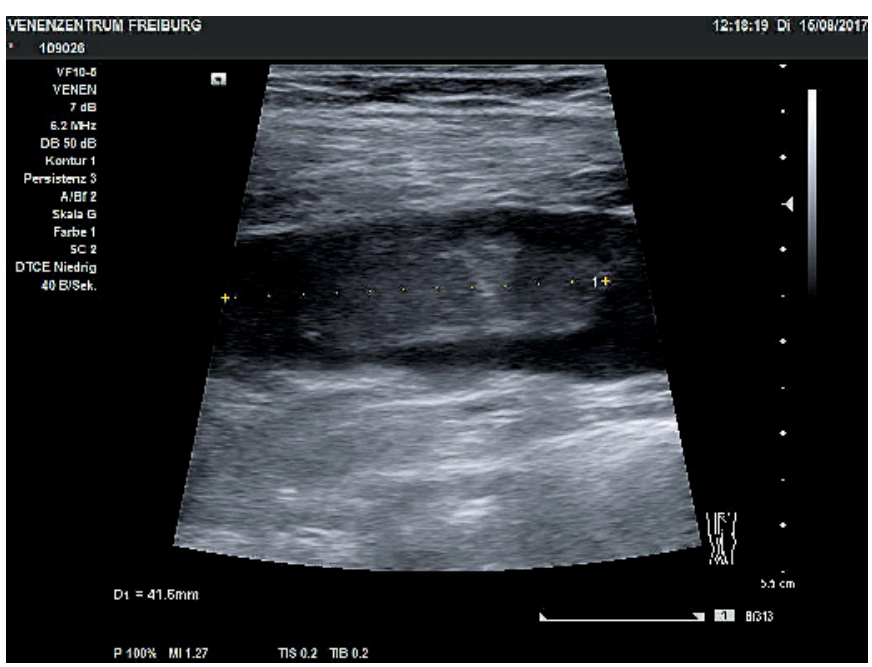

Abb. 12 Patient 6, PATE III links zehn Tage nach Laser VSM und VSAA links sowie der VSM rechts (Rezidiv). Eine Miniphlebektomie oder Schaumsklerosierung wurde nicht durchgeführt. Eine orale Antikoagulation (OAK) mit Rivaroxaban therapeutisch wurde eingeleitet. nicht erforderlich. Ausnahmen stellen zapfenartige Appositionsthromben dar, welche die Hämodynamik wesentlich beeinflussen könnten. Diese werden von den Autoren immer (auch bei Einengung des Venenlumens $<25 \%$ ) therapeutisch mit NMH behandelt.

Ab PATE II empfehlen die Autoren eine therapeutische Antikoagulation bis zum Verschwinden des Appositionsthrombus. Eine Nachkontrolle ist ebenfalls innerhalb von zwei Wochen indiziert.

Dies sind lediglich Empfehlungen. Eine Ausdehnung der Therapie eines PATE, an- gepasst an das individuelle Risikoprofil des Patienten, bleibt selbstverständlich dem behandelnden Arzt vorbehalten.

Diskussion der modifizierten PATEEinteilung und der Therapieempfehlungen:

Im Venenzentrum Freiburg traten im Jahr 201738 (2,19\%) PATE nach Laser bzw. 9 (1,65\%) PATE nach segmentaler Raidofrequenztherapie (RFA) (Closure Fast ${ }^{\circledR}$ ), bei insgesamt 1732 bzw. 543 durchgeführten Eingriffen, auf, 2015 waren es lediglich 7 (0,42\%) nach Laser bzw. $4(0,59 \%)$ nach RFA ( Tab. 1, $\gg$ Tab, 2, $\gg$ Tab. 3).
Die Differenzen bezüglich der Anzahl an aufgetretenen PATE nach Laser bzw. RFA von 2015-2017 in unserem Zentrum können durch die Anzahl an postoperativen duplexsonographischen Nachkontrollen erklärt werden. 2017 wurden alle Patienten zu einer Nachkontrolle nach ca. 7-14 Tagen einbestellt, 2016 wurde diese routinemäßige Nachschau nur bei ca. 50\% der Patienten durchgeführt, 2015 waren die Patienten erst nach ca. 6 Wochen wieder vorstellig, so dass sich einige PATE wieder rückgebildet haben können. Diesen Verdacht legt auch die aktuelle Literatur 


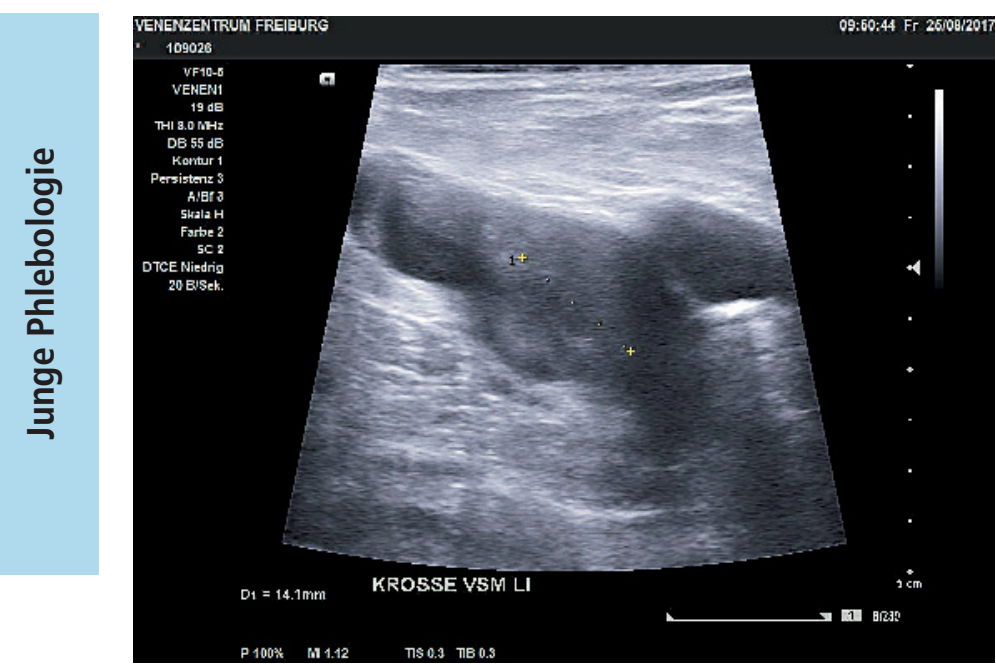

Abb. 13 Patient 6, nach zehntägiger Therapie mit Rivaroxaban therapeutisch, der Thrombus füllte weiterhin mehr als 50\% der Vena femoralis communis (VFC) aus. Die OAK mit Rivaroxaban therapeutisch wurde fortgesetzt.

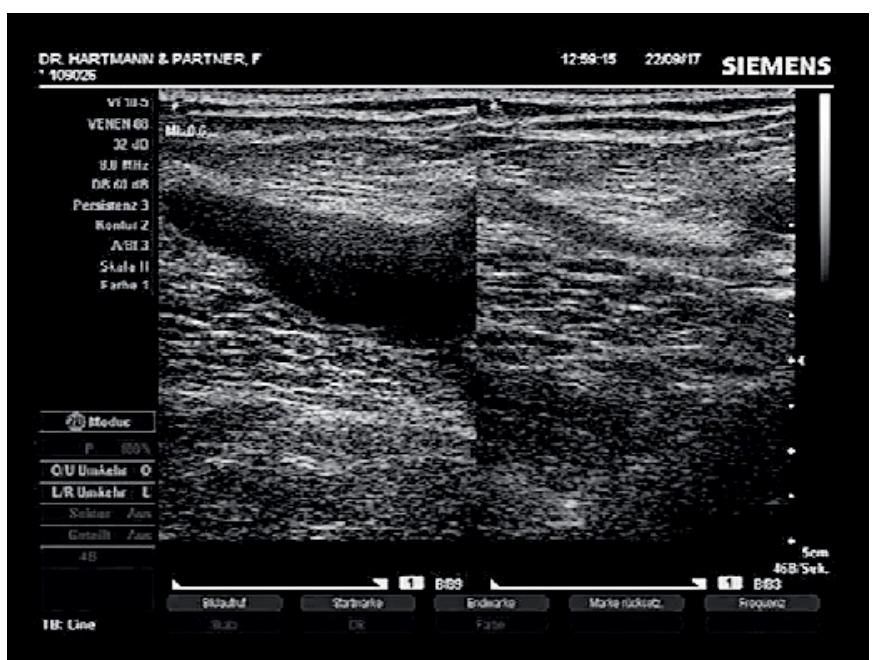

Abb. 14 Patient 6, Nachkontrolle nach 39 tägiger Therapie mit Rivaroxaban therapeutisch. Die VFC war wieder frei durchgängig, Rivaroxaban wurde abgesetzt.

Tab. 1 Anzahl (n) an Appositionsthromben als Komplikation endovenöser Katheterverfahren im Venenzentrum Freiburg im Jahr 2017

\begin{tabular}{l|c|c|c|}
\hline & Gesamt $\mathbf{n}(\%)$ & Laser $\mathbf{n}(\%)$ & RFA $\mathbf{n}(\%)$ \\
\hline Eingriffe & 2275 & 1732 & 543 \\
\hline PATE gesamt & $47(2,06)$ & $38(2,19)$ & $9(1,65)$ \\
\hline PATE I & $22(0,97)$ & $17(0,98)$ & $5(0,92)$ \\
\hline PATE II & $19(0,84)$ & $18(1,04)$ & $1(0,18)$ \\
\hline PATE III & $5(0,22)$ & $2(0,12)$ & $3(0,55)$ \\
\hline PATE IV & $1(0,04)$ & $1(0,06)$ & 0 \\
\hline
\end{tabular}

Tab. 2 Anzahl (n) an Appositionsthromben als Komplikation endovenöser Katheterverfahren im Venenzentrum Freiburg im Jahr 2016.

\begin{tabular}{|l|c|c|c|}
\hline & Gesamt $\mathbf{n}(\%)$ & Laser $\mathbf{n}(\%)$ & RFA $\mathbf{n}(\%)$ \\
\hline Eingriffe & 1911 & 1238 & 673 \\
\hline PATE gesamt & $32(1,67)$ & $17(1,37)$ & $13(1,93)$ \\
\hline PATE I & $9(0,47)$ & $4(0,32)$ & $5(0,74)$ \\
\hline PATE II & $17(0,89)$ & $10(0,80)$ & $6(0,89)$ \\
\hline PATE III & $4(0,20)$ & $2(0,16)$ & $1(0,15)$ \\
\hline PATE IV & $2(0,20)$ & $1(0,08)$ & $1(0,15)$ \\
\hline
\end{tabular}

Tab. 3 Anzahl (n) an Appositionsthromben als Komplikation endovenöser Katheterverfahren im Venenzentrum Freiburg im Jahr 2015

\begin{tabular}{l|c|c|c|}
\hline & Gesamt $\mathbf{n}(\%)$ & Laser $\mathbf{n}(\%)$ & RFA $\mathbf{n}(\%)$ \\
\hline Eingriffe & 1635 & 831 & 804 \\
\hline PATE gesamt & $7(0,42)$ & $3(0,36)$ & $4(0,59)$ \\
\hline PATE I & $3(0,18)$ & $1(0,12)$ & $2(0,29)$ \\
\hline PATE II & $3(0,18)$ & $2(0,24)$ & $1(0,12)$ \\
\hline PATE III & 0 & 0 & 0 \\
\hline PATE IV & $1(0,06)$ & 0 & $1(0,12)$ \\
\hline
\end{tabular}

nahe. Hier zeigen sich Progressionsraten eines unbehandelten EHIT I-II (= modifizierter PATE I-II) von lediglich ca. 3\% (5, 6).

Die Mehrzahl der PATE bilden sich sicherlich in den Wochen nach der endovenösen Therapie zurück, hier insbesondere PATE 1, wenn es sich um eine kleinere Vorwölbung handelt. Daher empfehlen die Autoren hier eine modifizierte Einteilung und abwartende Therapie bei einer Einengung des Lumens der tiefen Vene von bis zu 25\% und regelmäßigen Kontrollen bis zur Rückbildung des Appositionsthrombus. Ab PA-
TE 2 jedoch oder zapfenartigen (fluktuierenden) Appositionsthromben besteht eher die Gefahr für eine Progredienz des Thrombus und / oder einer Lungenembolie und daher empfehlen die Autoren ab PATE 2 (im Gegensatz zum internationalen Gebrauch) eine therapeutische Antikoagulation bis zur Rückbildung des Thrombus.

Die prophylaktische postoperative Gabe von $\mathrm{NMH}$ haben wir an das jeweilige Risikoprofil des Patienten angepasst. Rückschlüsse, ob eine längerfristige postoperative NMH-Gabe die Entstehung eines PATE verhindern kann, sollten deshalb aus unseren Daten nicht gezogen werden.

Therapeutisch war in einigen Fällen eine mehrwöchige Therapie mit NMH notwendig, in einigen Fällen konnte die Antikoagulation bereits nach zehn Tagen abgesetzt werden. ( $>$ Tab. 4) Dies unterstreicht die Notwendigkeit von duplexsonographischen Verlaufskontrollen in regelmäßigen Abständen bis zur vollständigen Rückbildung des Appositionsthrombus.

2017 traten in unserem Zentrum erfreulicherweise keine Thrombosen nach endovenösen Katheterverfahren auf . 2015 

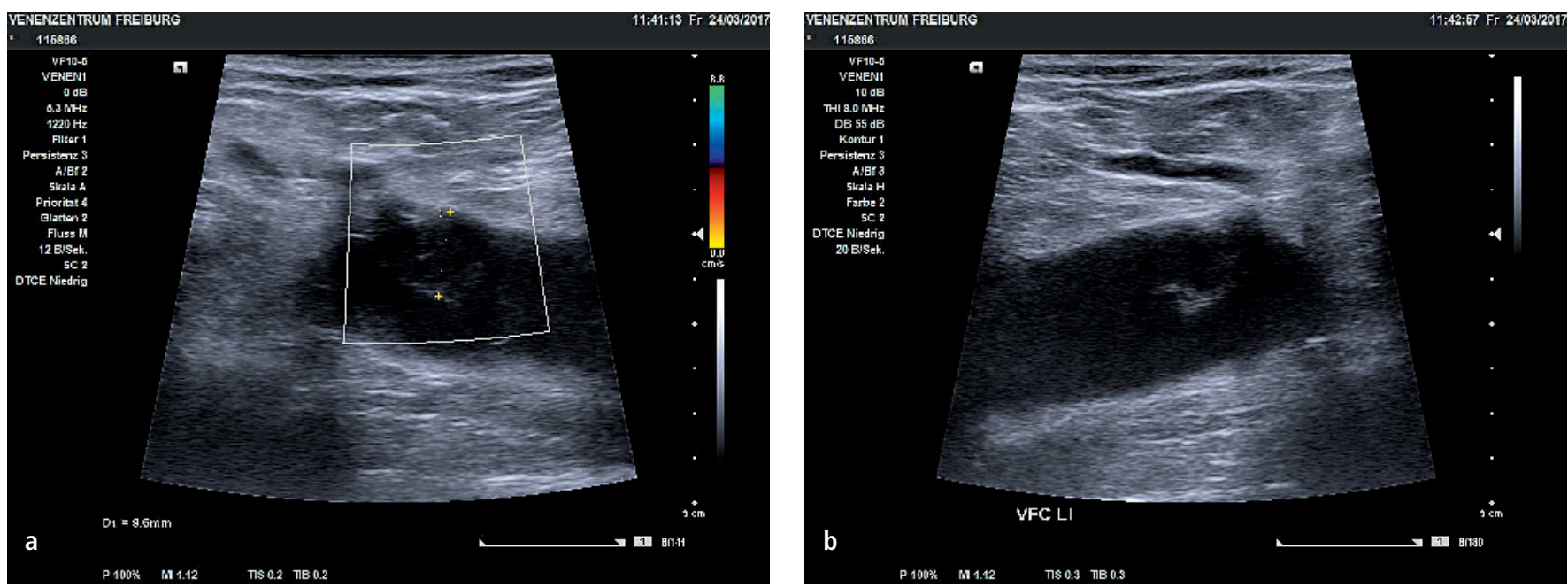

Abb. 15 a und b: Patient 7, PATE III links zehn Tage nach Laser der VSM links kombiniert mit einer Miniphlebektomie und Schaumsklerosierung. Eine gewichtsadaptierte Therapie mit Tinzaparin wurde eingeleitet.

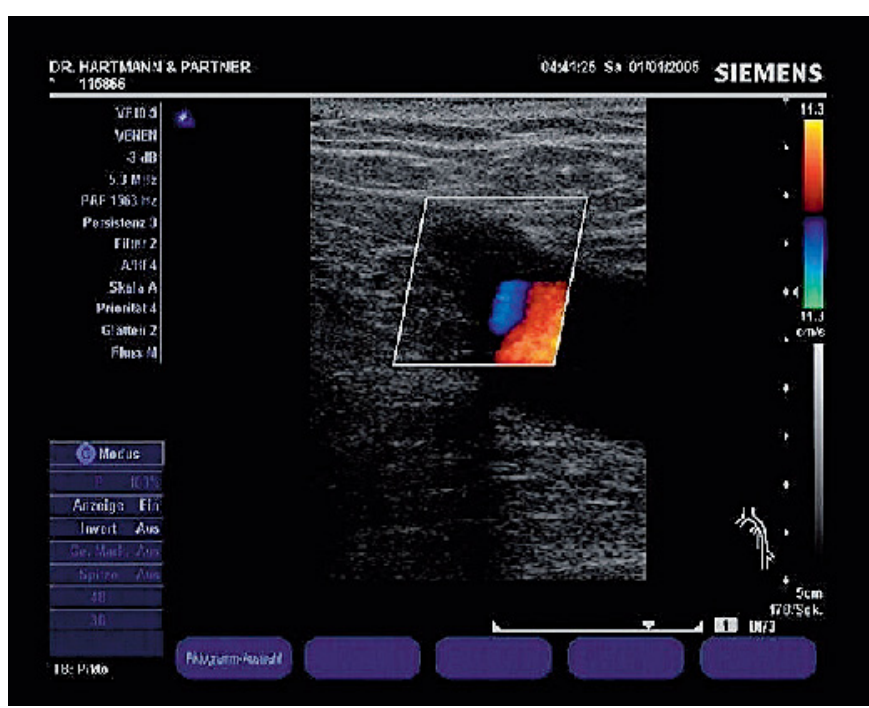

Abb. 16 Patient 7, Nachkontrolle nach zehntägiger Therapie mit Tinzaparin gewichtsadaptiert. Der Thrombus war nicht mehr darstellbar, Tinzaparin wurde nach insgesamt 10 Tagen abgesetzt.

wurde ein Patient mit TVT nach endovenösem Katheterverfahren vorstellig, 2016 waren zwei Patienten betroffen. ( $>$ Tab. 5) Bei zwei Patienten fanden duplexsonographische Nachuntersuchungen erst nach vier bzw. acht Wochen statt. Ein Patient war bereits bei einer Kontrolle am zehnten postoperativen Tag mit Thrombose vorstellig.

Zwei der drei betroffenen Patienten entwickelten außerdem eine Lungenembolie und hatten auch bei den nachfolgenden Kontrollen schwerwiegende postthrombotische Veränderungen mit tiefer Leitvene-
Abb. 18

Patient 8, Nach siebenwöchiger Therapie mit Rivaroxaban ist die Vene wieder frei durchgängig. Die orale Antikoagulation wurde, der leitliniengerechten Therapie einer TVT entsprechend, für drei Monate durchgeführt.

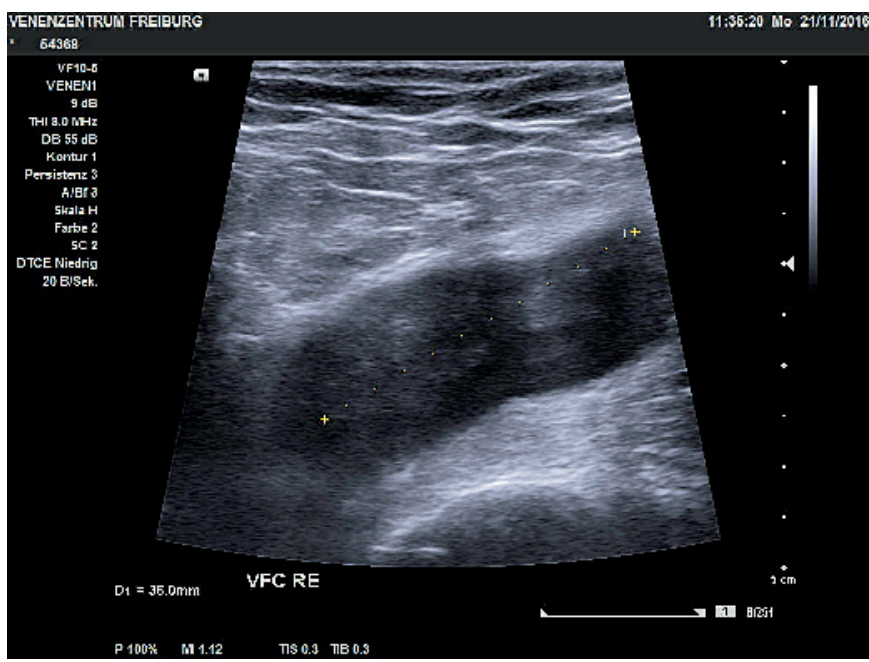

Abb. 17 Patient 8, PATE IV rechts (vollständiger kurzstreckiger Verschlus der VFC) zehn Tage nach Venenkleber (VenaSeal ${ }^{\odot}$ ) der VSM rechts kombiniert mit einer Miniphlebektomie und Schaumverödung. Eine OAK mit Rivaroxaban wurde eingeleitet.

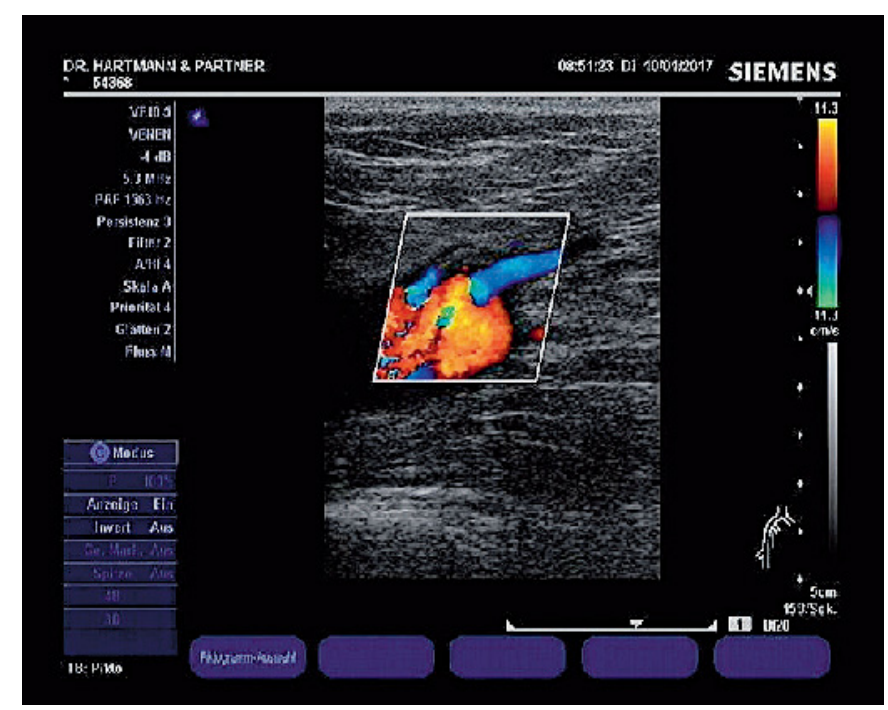


Tab. 4 Risikoprofil der Patienten mit Entwicklung eines PATE nach endovenösem Katheterverfahren von 2015-2017 im Venenzentrum Freiburg.

\begin{tabular}{|c|c|c|c|c|}
\hline & & 2015 & 2016 & 2017 \\
\hline \multicolumn{5}{|l|}{ PATE I } \\
\hline & Weiblich (\%) & 0 & $6(66,7)$ & $11(50)$ \\
\hline & Männlich (\%) & $3(100)$ & $3(33,3)$ & $11(50)$ \\
\hline & BMI & $32 \pm 0$ & $33 \pm 6$ & $26 \pm 5$ \\
\hline & Laser (\%) & $1(33,3)$ & 0 & $17(77,3)$ \\
\hline & RFT (\%) & $2(66,7)$ & $4(44,4)$ & $5(22,7)$ \\
\hline & Miniphlebektomie (\%) & $3(100)$ & $5(55,6)$ & $18(81,8)$ \\
\hline & VSM (\%) & $3(100)$ & $9(100)$ & $19(86,4)$ \\
\hline & VSP (\%) & 0 & 0 & $2(9,0)$ \\
\hline & $\begin{array}{l}\text { VSAA mitbehandelt } \\
(\%)\end{array}$ & 0 & 0 & $4(18,2)$ \\
\hline & Eingriff beidseits (\%) & $1(33,3)$ & $4(44,4)$ & $8(36,7)$ \\
\hline & Heparin post OP (Tage) & $6 \pm 0$ & $11 \pm 6$ & $6 \pm 7$ \\
\hline & NMH Therapie (Tage) & $9 \pm 2$ & $13 \pm 14$ & $8 \pm 8$ \\
\hline \multicolumn{5}{|l|}{ PATE II } \\
\hline & Weiblich (\%) & $1(33,3)$ & $5(27,8)$ & $8(42,1)$ \\
\hline & Männlich (\%) & $2(66,7)$ & $13(72,2)$ & $11(57,9)$ \\
\hline & BMI & & $29 \pm 3$ & $28 \pm 4$ \\
\hline & Laser (\%) & $2(66,6)$ & $11(61,1)$ & $18(94,7)$ \\
\hline & RFT (\%) & $1(33,3)$ & $6(33,3)$ & $1(5,3)$ \\
\hline & Andere & & $1(5,6)$ & \\
\hline & Miniphlebektomie (\%) & $1(33,3)$ & $11(61,1)$ & $11(57,9)$ \\
\hline & VSM (\%) & $3(100)$ & $15(83,3)$ & $17(89,5)$ \\
\hline & VSP (\%) & $1(33,3)$ & $3(16,7)$ & $1(5,3)$ \\
\hline & $\begin{array}{l}\text { VSAA mitbehandelt } \\
(\%)\end{array}$ & 0 & $4(22,2)$ & $5(26,3)$ \\
\hline & Eingriff beidseits (\%) & $1(33,3)$ & $3(16,7)$ & $8(42,1)$ \\
\hline & Heparin post OP (Tage) & $6 \pm 0$ & $8 \pm 5$ & $7 \pm 10$ \\
\hline & NMH Therapie (Tage) & $16 \pm 8$ & $23 \pm 18$ & $19 \pm 8$ \\
\hline
\end{tabular}

\begin{tabular}{|c|c|c|c|c|}
\hline & & 2015 & 2016 & 2017 \\
\hline \multicolumn{5}{|l|}{ PATE III } \\
\hline & Weiblich (\%) & 0 & $3(75)$ & $2(40)$ \\
\hline & Männlich (\%) & 0 & $1(25)$ & $3(60)$ \\
\hline & BMI & & $25 \pm 3$ & $26 \pm 2$ \\
\hline & Laser (\%) & 0 & $2(50)$ & $2(40)$ \\
\hline & RFT (\%) & 0 & $1(25)$ & $3(60)$ \\
\hline & Andere & & $1(25)$ & \\
\hline & Miniphlebektomie (\%) & 0 & $3(75)$ & $3(60)$ \\
\hline & VSM (\%) & 0 & $4(100)$ & $3(60)$ \\
\hline & VSP (\%) & 0 & 0 & $2(40)$ \\
\hline & $\begin{array}{l}\text { VSAA mitbehandelt } \\
(\%)\end{array}$ & 0 & 0 & 0 \\
\hline & Eingriff beidseits (\%) & 0 & 0 & $1(20)$ \\
\hline & Heparin post OP (Tage) & 0 & $12 \pm 9$ & $6 \pm 6$ \\
\hline & NMH Therapie (Tage) & 0 & $74 \pm 41$ & $17 \pm 5$ \\
\hline \multicolumn{5}{|l|}{ PATE IV } \\
\hline & Weiblich (\%) & 0 & $1(50)$ & $1(100)$ \\
\hline & Männlich (\%) & 1 & $1(50)$ & 0 \\
\hline & BMI & & $32 \pm 3$ & \\
\hline & Laser (\%) & 0 & $1(50)$ & $1(100)$ \\
\hline & RFT (\%) & $1(100)$ & $1(50)$ & 0 \\
\hline & Miniphlebektomie (\%) & $1(100)$ & $2(100)$ & 0 \\
\hline & VSM (\%) & $1(100)$ & $1(50)$ & $1(100)$ \\
\hline & VSP (\%) & $1(100)$ & $1(50)$ & 0 \\
\hline & $\begin{array}{l}\text { VSAA mitbehandelt } \\
(\%)\end{array}$ & 0 & 0 & $1(100)$ \\
\hline & Eingriff beidseits (\%) & $1(100)$ & $1(50)$ & 0 \\
\hline & Heparin post OP (Tage) & $6 \pm 0$ & $17 \pm 15$ & $2 \pm 0$ \\
\hline & NMH Therapie (Tage) & $84 \pm 0$ & dauerhaft & $30 \pm 0$ \\
\hline
\end{tabular}

ninsuffizenz, so dass hier eine dauerhafte orale Antikoagulation indiziert war. Eine TVT (kein PATE IV!), welche bereits 10 Tage nach der Intervention auftritt, sollte als direkte Nebenwirkung der Therapie klassifiziert werden und tritt laut Literatur in $0,6 \%-1 \%$ der behandelten Venen auf (3, 7).

Schlussfolgernd konnten wir 2017 durch regelmäßige Nachkontrollen und

\begin{tabular}{l|l|l|l|}
\hline Jahr & TVTs gesamt & $\begin{array}{l}\text { TVTs n (\%) nach } \\
\text { Laser }\end{array}$ & $\begin{array}{l}\text { TVTs n (\%) nach } \\
\text { VNUS }\end{array}$ \\
\hline 2017 & 0 & 0 & 0 \\
\hline 2016 & $2(0,1)$ & $1(0,08)$ & $1(0,14)$ \\
\hline 2015 & $1(0,06)$ & 0 & $1(0,12)$ \\
\hline
\end{tabular}

Tab. 5

Anzahl (n) an TVTs nach endovenösen Katheterverfahren im Venenzentrum Freiburg von 2015-2017. konsequente Therapie der PATE vermutlich TVTs verhindern. Allerdings hat die Auswertung der Daten gezeigt, dass PATE in geringer Ausprägung (PATE 1) nicht zwingend immer behandelt werden müssen und eine Rückbildung auch ohne NMH stattfindet.

Um auch in Zukunft einen guten und sicheren Standard bezüglich der postoperativen Versorgung von endovenös behandelten Patienten zu entwickeln sind sicherlich weitere pro-, und retrospektive Studien notwendig. 


\section{Interessenkonflikt}

Die Autoren geben an, dass kein Interessenkonflikt besteht.

\section{Ethische Richtlinien}

Die Arbeit wurde unter Einhaltung aller nationaler Richtlinien sowie der aktuellen Deklaration von Helsinki erstellt.

\section{Literatur}

1. Hartmann K, Alm J, Breu F-X, Maurins U, Pannier F, Reich-Schupke S. Endovenöse Verfahren, minimalinvasive Therapie der Varikosis. Stuttgart: Schattauer 2015, 164-166.

2. Korepta LM, Watson JJ, Mansour MA, Chambers CM, Cuff RF, Slaikeu JD, Wong PY. Outcomes of a single-center experience with classification and treatment of endothermal heat-induced thrombosis after endovenous ablation. J Vasc Surg Venous Lmphat Disord 2017; 5(3): 332-228.

3. Marsh P, Price BA, Holdstock J, Harrison C, Whiteley MS. Deep vein thrombosis (DVT) after ve- nous thermoablation techniques: rates of endovenous heat-induced thrombosis (EHIT) and classical DVT after radiofreqency and endovenous laser ablation in a single centre. Eur J Vasc Endovasc Surg 2010; 404(4): 521-527.

4. Mozes G, Kalra M, Carmo M, Swenson L, Gloviczik P. Extension of saphenous thrombus into the femoral vein: a potential complication of new endovenous ablation thechniques. J Vasc Surg 2005; 41: 130-135.

5. Sufian S, Arnez A, Labropoulos N, Lakhanpal S Incidence, progression, and risk factors for endovenous heat-induced thrombosis after radiofrequency ablation. J Vasc Surg Venous Lmphat Disord 2013; 1(2): 159-164. 